\title{
Detección de tornillos prominentes en la articulación radiocarpiana mediante la proyección tangencial del escafoides dorsal
}

\section{Detection of Protruding Screws in the Radiocarpal Joint Using the "Scaphoid Skyline View"}

\author{
Juan Manuel Breyer ${ }^{1,2}$ Alfonso Pérez ${ }^{1,3(0)}$ Pamela Vergara ${ }^{1,3}$ Pedro Ruiz ${ }^{4}$
}

\footnotetext{
${ }^{1}$ Hospital del Trabajador, Región Metropolitana, Santiago, Chile

${ }^{2}$ Clínica Alemana de Santiago, Región Metropolitana, Santiago, Chile

${ }^{3}$ Clínica Las Condes, Región Metropolitana, Santiago, Chile

${ }^{4}$ Hospital Puerto Montt Dr. Eduardo Schütz Schroeder, Puerto Montt, Chile
}

Rev Chil Ortop Traumatol 2021;62:27-33.

\begin{abstract}
Address for correspondence Juan Manuel Breyer, Clínica Alemana de Santiago, avenida Vitacura 5.951, Vitacura, Región Metropolitana, Santiago, Chile (e-mail: jmbreyer@hotmail.com).
\end{abstract}

\section{Resumen}

Palabras claves

- complicaciones

- escafoides

- osteosíntesis

- fijación de tornillos

- proyección radiográfica

- fluoroscopía
Introducción Una de las complicaciones de la osteosíntesis retrógrada del escafoides es la protrusión del tornillo en la articulación radiocarpiana, dada la limitada visualización intraoperatoria del polo proximal del escafoides con las proyecciones tradicionales.

Objetivo Evaluar la capacidad de una nueva proyección radiológica, la proyección "tangencial del escafoides dorsal", o TED, para identificar tornillos prominentes radiocarpianos durante la osteosíntesis retrógrada del escafoides con tornillos canulados.

Materiales y Métodos Estudio cadavérico en muñecas frescas congeladas. Se introdujo en el escafoides un tornillo canulado con técnica retrógrada estándar.

La proyección TED fue evaluada en 5 muñecas, con angulaciones del antebrazo de $15^{\circ}$, $30^{\circ}$ y $45^{\circ}$ para definir la mejor visualización del polo proximal del escafoides y del tornillo.

Se comparó la capacidad para identificar la prominencia del tornillo en el polo proximal de la proyección TED de $30^{\circ}$ con 5 proyecciones tradicionales de escafoides en 9 muñecas. El tornillo se posicionó a nivel de la superficie del escafoides, y luego se avanzó a intervalos de $0,5 \mathrm{~mm}$ bajo visualización directa por artrotomía dorsal. Tras cada intervalo, se repitieron todas las proyecciones para determinar su capacidad de detectar tornillos prominentes en el escafoides.

Resultados La mejor visualización del polo proximal del escafoides se logró con la proyección TED de $30^{\circ}$. Al comparar la proyección TED de $30^{\circ}$ y las tradicionales, con la TED se logró identificar tornillos prominentes a $0,8 \mathrm{~mm}$ promedio, seguida por la received

July 27,2020

accepted

January 21, 2021
DOI https://doi.org/

$10.1055 / \mathrm{s}-0041-1728733$. ISSN 0716-4548.
(C) 2021. Sociedad Chilena de Ortopedia y Traumatologia. All rights reserved.

This is an open access article published by Thieme under the terms of the Creative Commons Attribution-NonDerivative-NonCommercial-License, permitting copying and reproduction so long as the original work is given appropriate credit. Contents may not be used for commercial purposes, or adapted, remixed, transformed or built upon. (https://creativecommons.org/ licenses/by-nc-nd/4.0/)

Thieme Revinter Publicações Ltda., Rua do Matoso 170, Rio de Janeiro, RJ, CEP 20270-135, Brazil 
proyección posteroanterior con cubitalización y extensión a $1.3 \mathrm{~mm}(p=0.014)$, con una alta precisión y correlación interobservador de estas proyecciones.

Conclusión La proyección TED demostró ser la más sensible para detectar tornillos prominentes en la articulación radiocarpiana. Su uso rutinario podría evitar complicaciones durante la osteosíntesis.

\section{Abstract}

\section{Keywords}

- complications

- scaphoid

- osteosynthesis

- screw fixation

- X-ray view

- fluoroscopy
Introduction One of the complications of the retrograde percutaneous scaphoid fixation is the protrusion of the screw in the radiocarpal joint due to the limited intraoperative visualization of the proximal pole of the scaphoid with the traditional radiographic views.

Objetive To evaluate the sensitivity of a novel radiographic view (the skyline scaphoid view, SSV) to detect screws protruding in the radiocarpal joint during the retrograde fixation of the scaphoid.

Materials and Methods We studied nine cadaverous fresh frozen wrists. A retrograde cannulated screw was inserted in the scaphoid.

To validate the SSV, 5 wrists were studied, comparing 3 forearm angulations $\left(15^{\circ}, 30^{\circ}\right.$ and $45^{\circ}$ ) to get the best visualization of the proximal pole and screw.

We compared the ability to identify the protrusion of the screw in the proximal pole of the $30^{\circ} \mathrm{SSV}$ with that of 5 standard scaphoid radiographic views in 9 wrists. The screw was positioned at the level of the surface of the scaphoid, and was sequentially protruded in $0.5 \mathrm{~mm}$ increments, with direct visualization of its tip through a dorsal capsulotomy. After each increment, all views were repeated to determine if they were able to detect screws projecting from the scaphoid.

Results The best visualization of the proximal pole of the scaphoid was found with the $30^{\circ} \mathrm{SSV}$. In the comparison of the $30^{\circ} \mathrm{SSV}$ and the standard views, with the SSV we were able to identify the protrusion of the screws at an average of $0.8 \mathrm{~mm}$, followed by the posterior-anterior view with ulnar deviation and extension at $1.3 \mathrm{~mm}(p=0.014)$, with high precision and interobserver agreement regarding these views.

Conclusion The SSV was the most sensitive view to detect protruding screws in the proximal pole of the scaphoid. Its routine use could avoid complications during osteosynthesis.

\section{Introducción y objetivo}

La osteosíntesis con tornillo canulado, sin cabeza, y retrógrado es una de las técnicas más utilizadas en el tratamiento de la fractura de escafoides. ${ }^{1}$ Dentro de los principios de tratamiento para lograr la mayor estabilidad de la fractura y consolidación de ésta, se describe que el tornillo debe ubicarse perpendicular al rasgo de fractura y tener el mayor largo possible. ${ }^{2}$

Para lograr este último punto durante la inserción retrógrada del tornillo, es fundamental la correcta visualización bajo fluoroscopía de la región dorsal y proximal del escafoides, ya que es en esta región donde se ubicará la punta del tornillo. Lo anterior puede ser difícil de lograr durante la cirugía debido a la anatomía del escafoides y la sobreposición de los otros huesos del carpo en la proyección lateral de muñeca. Debido a esto, existe el riesgo de que un tornillo largo protruya en la articulación radiocarpiana.

Existen una serie de proyecciones radiológicas que tradicionalmente se utilizan tanto en el diagnóstico de la fractura como en control radiográfico intraoperatorio de la osteosíntesis de escafoides. Entre ellas, las proyecciones oblicuas permitirían una mejor visualización del polo proximal del escafoides, y, por lo tanto, también permitirían prevenir tornillos que protruyan a la articulación. Sin embargo, su sensibilidad está lejos de ser óptima, pues puede haber tornillos prominentes hasta $1 \mathrm{~mm}$ que no son detectados. ${ }^{3}$

Con el fin de mejorar la visualización del polo proximal e identificar tornillos de escafoides protruyentes radiocarpianos, proponemos una nueva proyección radiológica intraoperatoria: la proyección "tangencial del escafoides dorsal", o TED.

Esta proyección corresponde a una modificación de la proyección tangencial para el radio dorsal propuesta por Jacob y Clay, ${ }^{6}$ la skyline view, utilizada para evaluar tornillos prominentes en el dorso del radio durante la osteosíntesis con placa volar del radio. ${ }^{4-6}$

Los objetivos de este estudio fueron estandarizar la técnica de la nueva proyección radiográfica TED, y evaluar su capacidad para detectar tornillos prominentes en el polo 
proximal del escafoides durante la osteosíntesis retrógrada de escafoides, comparándola con otras cinco proyecciones.

\section{Material y Métodos}

Estudio experimental en piezas cadavéricas. Se utilizaron nueve muñecas frescas congeladas. Se comprobó, bajo fluoroscopía (posteroanterior y lateral), la ausencia de fracturas y/o artrosis radio o mediocarpiana.

Mediante un miniabordaje volar, se introdujo un tornillo canulado Acutrak2 Mini (Acumed, Hillsboro, OR, EEUU) de acuerdo a la técnica estándar de osteosíntesis volar retrógrada del escafoides con apoyo fluoroscópico, posicionando la punta del tornillo lo más cerca posible de la cortical proximal-dorsal del escafoides. ${ }^{7}$

\section{Proyección TED}

La imagen se obtiene con el intensificador de imágenes en posición vertical, orientado axial y tangencialmente al dorso del escafoides y carpo. El antebrazo se posiciona en supinación, la muñeca, en flexión máxima, y el codo, en semiflexión (-Figura 1).

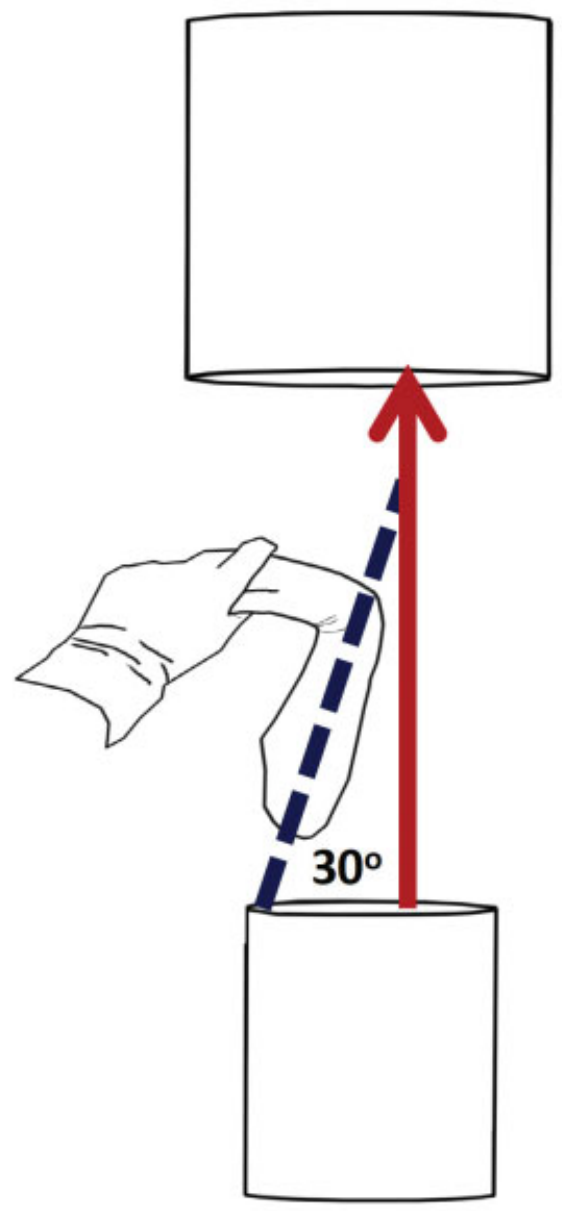

Fig. 1 Posicionamiento del intensificador de imágenes y de la extremidad superior, con $30^{\circ}$ de angulación del antebrazo, para la proyección tangencial del escafoides dorsal (TED).
Para estandarizar la técnica de la proyección TED, se realizó un estudio en 5 muñecas, en el que se realizó la proyección TED con angulaciones de $15^{\circ}, 30^{\circ}$ y $45^{\circ}$ del antebrazo respecto al eje vertical del rayo, evaluando la visualización del tornillo y el polo proximal del escafoides.

\section{Comparación de Proyecciones Radiográficas}

En 9 muñecas cadavéricas, se determinó la ubicación de la punta del tornillo dentro o fuera del escafoides, utilizando proyección TED de $30^{\circ}$ y 5 proyecciones tradicionales: posteroanterior (PA), posteroanterior cubitalizada (PAC), posteroanterior cubitalizada extendida (PACE), oblicua pronada (OP), y lateral (L) ${ }^{3}$ (- Figura 2).

La serie de estas seis proyecciones se realizó inicialmente con la punta del tornillo a nivel de la superficie articular. Posteriormente, se avanzó el tornillo secuencialmente $0,5 \mathrm{~mm}$, protruyendo el polo proximal del escafoides. Luego de cada avance, se repitieron las 6 proyecciónes (TED de $30^{\circ}$ y las 5 tradicionales), hasta que fue evidente que la punta del tornillo protruía del polo proximal en todas las proyecciones.

La ubicación de la punta del tornillo y la protrusión de éste se confirmaron bajo visión directa del polo proximal, a través de una artrotomía dorsal y la medición del tornillo con un cáliper digital. (- Figura $\mathbf{3}$ ).

Las imágenes fueron almacenadas digitalmente en formato Imagenología Digital y Comunicaciones en Medicina (Digital Imaging and Communications in Medicine, DICOM) para posterior evaluación. Las imágenes fueron recodificadas y ordenadas de forma aleatoria para la evaluación ciega por tres cirujanos de mano y un traumatólogo general. Después de una etapa de estandarización de los observadores, cada evaluador calificó deforma independiente cada una de las radiografías, definiendo si el tornillo se encontraba dentro o fuera del escafoides. En aquellos casos discordantes, la calificación fue consensuada.

La distancia mínima promedio (en $\mathrm{mm}$ ) a la cual fue posible detectar tornillos largos en las distintas proyecciones radiográficas fue comparada utilizando análisis de la varianza en una dirección (one-way analysis of variance [ANOVA]). Previo a la ANOVA, se realizó la prueba de Kolmogorov-Smirnoff para confirmar la distribución normal de los distintos grupos (proyecciones radiográficas). No se realizaron pruebas post hoc para detectar diferencias entre grupos debido a lo reducido de la muestra. La precisión de las distintas proyecciones para detectar tornillos prominentes se evaluó por curva característica operativa del receptor (receiver operating characteristic, ROC). La variabilidad interobservador se evaluó mediante el coeficiente de correlación intraclase. Se estableció el nivel de significancia estadística con $p<0,05$. El análisis estadístico se realizó con el programa Stata (StataCorp, College Station, TX, EEUU), versión 16.

\section{Resultados}

Para la estandarización de la técnica radiográfica para la proyección TED y la comparación de la proyección con $15^{\circ}$, $30^{\circ}$ y $45^{\circ}$ de angulación del antebrazo, no se observaron diferencias estadísticamente significativas en cuanto a la 


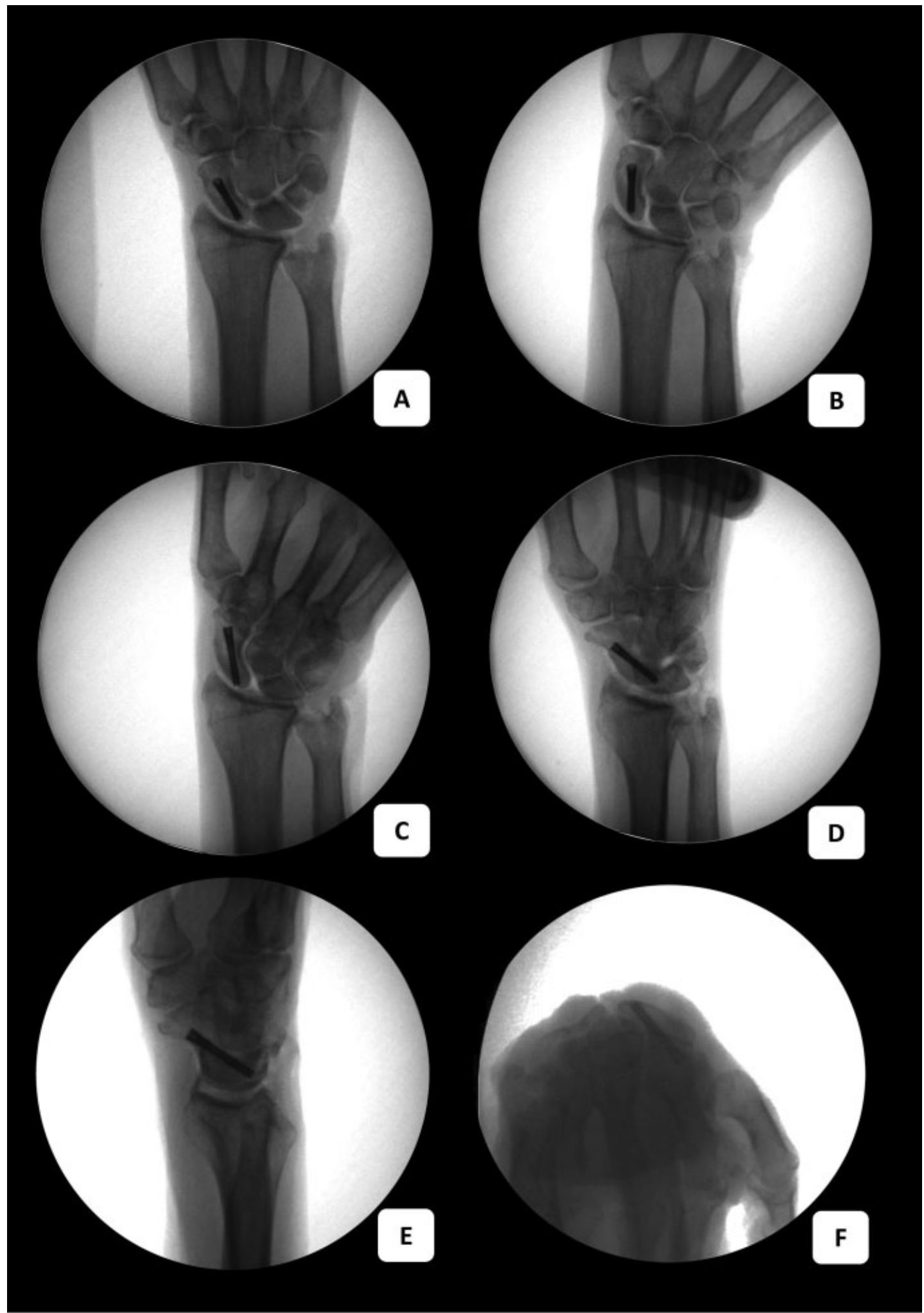

Fig. 2 Proyecciones radiográficas estudiadas. (A) Posteroanterior (PA); (B) posteroanterior cubitalizada (PAC); (C) posteroanterior cubitalizada extendida (PACE); (D) oblicua pronada (OP); (E) lateral (L); y (F) tangencial del escafoides dorsal (TED).

sensibilidad para detectar tornillos prominentes, y se identificaron tornillos prominentes con $0,8 \mathrm{~mm}$ en la TED de $30^{\circ}$ (desviación estándar [DS]: 0,3 mm), 0,94 $\mathrm{mm}$ en la TED de $45^{\circ}$ (DS: $0,94 \mathrm{~mm}$ ), y $1,0 \mathrm{~mm}$ en la TED de $15^{\circ}$ (DS: $0,66 \mathrm{~mm}$ ). Dado que durante estas pruebas se observó una subjetiva mejor visualización del polo proximal por los investigadores con la proyección TED de $30^{\circ}$ y la tendencia a ser mejor que las otras técnicas, fue esta la proyección utilizada para la comparación de la TED con las proyecciones tradicionales. 

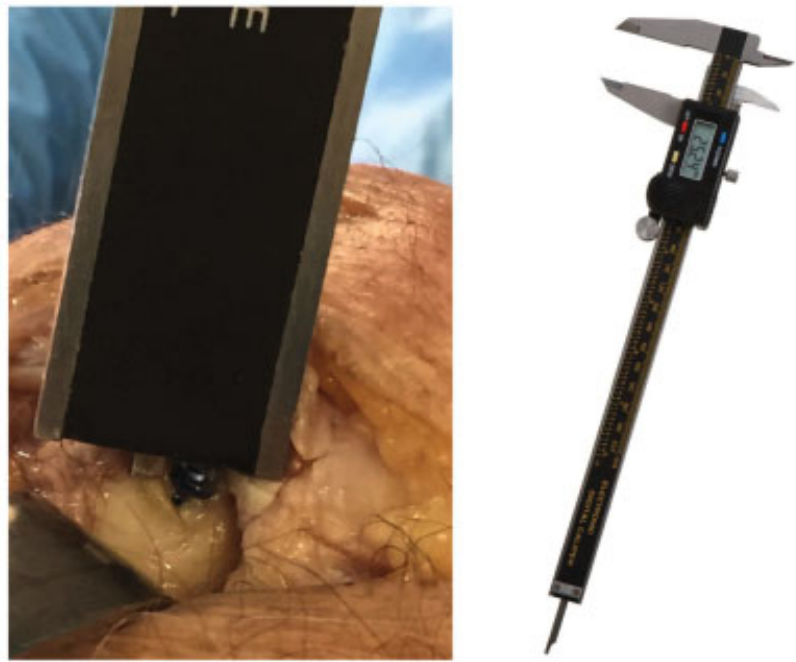

Fig. 3 Medición de la longitud del tornillo prominente en articulación radiocarpiana con cáliper digital.

Al evaluar y comparar la capacidad para identificar tornillos prominentes de TED y las proyecciones tradicionales, con la TED se pudo detectar tornillos protruyentes en promedio a $0,8 \mathrm{~mm}$ (DS 0,3 mm), seguida por la proyección PACE, a 1,3 $\mathrm{mm}$ (DS: 0,6 mm) (-Tabla 1).

De manera similar, entre las 6 proyecciones estudiadas, la TED mostró la mayor precisión, con un área bajo la curva ROC de 0,92 (-Tabla 2).

En cuanto a la correlación interobservador de las distintas proyecciones, todas demostraron tener una muy buena correlación, con un coeficiente de correlación intraclase mayor a 0,75 (-Tabla 3 ).

\section{Discusión}

Diversos estudios clínicos y biomecánicos ${ }^{1,2}$ han confirmado los beneficios en la osteosíntesis retrógrada del escafoides, la cual requiere de un posicionamiento y largo precisos del tornillo para favorecer la estabilidad y consolidación de la fractura.

Una seria complicación de la osteosíntesis retrógrada es la protrusión del tornillos en el polo proximal del escafoides, quedando de esta manera la punta del tornillo prominente a la articulación radiocarpiana, con el consiguiente daño del cartílago articular del radio, pérdida de función, y dolor. ${ }^{8}$

Las proyecciones radiográficas intraoperatorias son fundamentales para el correcto posicionamiento del tornillo de osteosíntesis. El problema es que, debido a la morfología del escafoides (curvo y cónico en el polo proximal), sumada a la sobreposición con los otros huesos del carpo y del radio, no se logra una adecuada definición del polo proximal del escafoides con las proyecciones radiográficas convencionales, pudiendo estar la punta del tornillo fuera del escafoides, sin ser observada por el cirujano. ${ }^{3,8}$

Un problema similar se puede observar en la osteosíntesis con placa volar en las fractura del radio distal, en las que, al evaluar el largo de los tornillos en la proyección lateral, el tubérculo de Lister puede impedir la correcta visualización de un tornillo largo y prominente en el dorso del radio. Para evitar este problema, Jacob y Clay ${ }^{6}$ describieron una proyección tangencial a la cortical dorsal del radio, denominada "proyección de horizonte dorsal del radio" o skyline view, que ha sido útil en identificar estos tornillos prominentes. ${ }^{4,6}$

En este estudio se presenta una modificación de la proyección skyline view de radio, en la que, aumentando la extensión del codo y así el ángulo entre el eje vertical del rayo $\mathrm{X}$ y el antebrazo (de los $15^{\circ}$ de la skyline de radio a $30^{\circ}$ ), se logra desproyectar el dorso del radio y visualizar de manera tangencial el polo proximal del escafoides, permitiendo evaluar tornillos protruyentes hacia la articulación radiocarpiana (-Figura 4 ).

En relación al mejor ángulo del antebrazo para realizar la proyección TED, si bien no hubo diferencias significativas en cuanto a la sensibilidad para detectar tornillos prominentes entre los $15^{\circ}, 30^{\circ}$ y $45^{\circ}$, encontramos una leve superioridad subjetiva de la TED de $30^{\circ}$ para visualizar el polo proximal del escafoides, por lo que la planteamos como la angulación ideal para su realización.

En cuanto a los resultados de este estudio, la proyección TED mostró tener una alta sensibilidad para detectar tornillos prominentes en el dorso del escafoides, siendo la más sensible de las proyecciones estudiadas, capaz de detectar tornillos largos a $0,8 \mathrm{~mm}$ de la superficie del polo proximal del escafoides en promedio. Además de lo anterior, la proyección

Tabla 1 Longitud promedio de detección de tornillos prominentes

\begin{tabular}{|l|l|l|l|l|}
\hline Proyección radiográfica & Protrusión & Desviación & \multicolumn{2}{l|}{$\begin{array}{l}\text { Intervalo de } \\
\text { confianza del 95\% }\end{array}$} \\
\cline { 3 - 5 } & & & Menor & Mayor \\
\hline Tangencial del escafoides dorsal & 0,8 & 0,3 & 0,5 & 1 \\
\hline Postero-anterior cubitalizada extendida & 1,3 & 0,6 & 0,7 & 1,9 \\
\hline Lateral & 1,3 & 1 & 0,3 & 2,2 \\
\hline Oblicua pronada & 1,4 & 0,9 & 0,4 & 2,3 \\
\hline Postero-anterior cubitalizada & 2,1 & 1 & 1 & 3,2 \\
\hline Postero-anterior & 2,2 & 0,9 & 1 \\
\hline
\end{tabular}

Nota: Longitud mínima promedio en la que las distintas proyecciones radiográficas detectaron tornillos prominentes. Análisis de la varianza de una vía (one-way ANOVA), correción de Welch $(p=0,014 ; \mathrm{F}=3,756)$. 
32 Proyección tangencial del escafoides dorsal Breyer y col

Tabla 2 Análisis por curva característica operativa del receptor de las distintas proyecciones radiográficas

\begin{tabular}{|l|l|l|l|l|}
\hline Proyección radiográfica & Área bajo la curva & \multicolumn{2}{l|}{$\begin{array}{l}\text { Intervalo de } \\
\text { confianza del 95\% }\end{array}$} \\
\cline { 2 - 5 } & & Menor & Mayor \\
\hline Tangencial del escafoides dorsal & & 0,83 & 1,00 & 0,001 \\
\hline Oblicua pronada & 0,92 & 0,62 & 0,93 & 0,06 \\
\hline Lateral & 0,77 & 0,57 & 0,90 & 0,02 \\
\hline Postero-anterior cubitalizada extendida & 0,73 & 0,54 & 0,89 & 0,03 \\
\hline Postero-anterior cubitalizada & 0,71 & 0,39 & 0,74 & 0,54 \\
\hline Posteroanterior & 0,56 & 0,50 & 0,88 & 0,08 \\
\hline
\end{tabular}

Tabla 3 Evaluación de la concordancia interobservador de las distintas proyecciones radiográficas

\begin{tabular}{|l|l|l|l|l|l|}
\hline Proyección Radiográfica & Coeficiente de & \multicolumn{2}{l|}{$\begin{array}{l}\text { Intervalo de } \\
\text { confianza del 95\% }\end{array}$} & \multirow{2}{*}{$\boldsymbol{F}$} \\
\cline { 2 - 5 } & intraclase & Menor & Mayor & \\
\cline { 2 - 5 } & 0,83 & 0,70 & 0,91 & 6,02 & $<0,001$ \\
\hline Pangencial del escafoides dorsal & 0,89 & 0,82 & 0,93 & 8,88 & $<0,001$ \\
\hline Posteroanterior cubitalizada & 0,95 & 0,91 & 0,97 & 18,21 & $<0,001$ \\
\hline Oblicua pronada & 0,91 & 0,85 & 0,95 & 11,48 & $<0,001$ \\
\hline Lateral & 0,75 & 0,58 & 0,87 & 4,06 & $<0,001$ \\
\hline Posteroanterior cubitalizada extendida & 0,93 & 0,88 & 0,96 & 14,51 & $<0,001$ \\
\hline
\end{tabular}

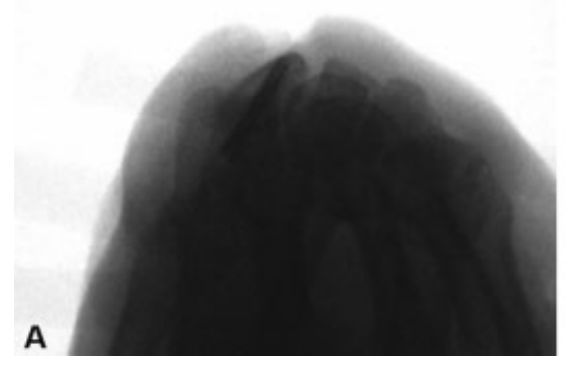

B

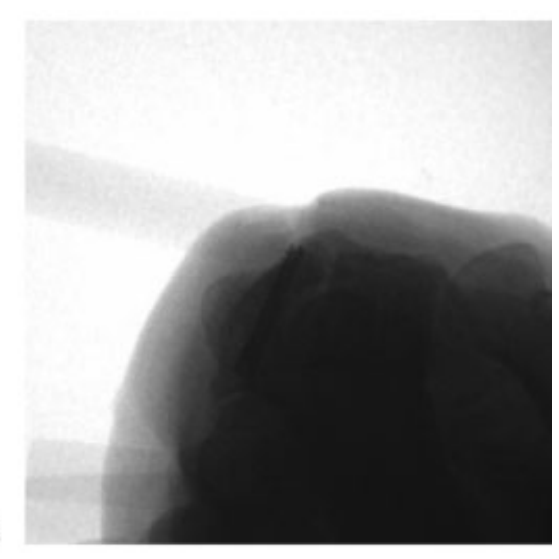

Fig. 4 Proyecciones tangenciales del escafoides dorsal (TEDs). (A) Tornillo intraóseo. (B) tornillo prominente fuera del escafoides.

TED demostró una elevada precisión, con una alta sensibilidad y baja proporción de falsos positivos (área bajo la curva ROC: 0,923 ) y una alta correlación interobservador.

En cuanto a la capacidad de las proyecciones tradicionales en identificar tornillos largos, nuestros resultados son similares a los descritos por Kim et al., ${ }^{3}$ quienes observaron que la proyección OP tuvo la mayor sensibilidad de las proyecciones tradicionales, con valores similares a los observados en nuestra serie $(1.4 \mathrm{~mm})$.

En base a la evaluación de la proyección TED de este estudio, a la sencillez del procedimiento, y lo complejo de tener un tornillo prominente, creemos que esta proyección debiera utilizarse de manera rutinaria en la práctica clínica, para reducir así las complicaciones de tornillos largos protruyentes. Se debe considerar que factores clínicos como el índice de masa corporal, la posición del antebrazo del paciente, y factores propios del trauma (edema, derrame articular etc.), podrían afectar la visión del escafoides con esta proyección.

\section{Conclusión}

La proyección TED es un método novedoso y sensible para la detección de tornillos que protruyen del polo proximal durante la osteosíntesis de escafoides con tornillo retrógrado, siendo altamente recomendable su uso rutinario clínico. 


\section{Conflicto de Intereses}

Los autores declaran no haber conflicto de intereses. Dr. Breyer relata apoyo no financiero de la empresa CLP, no relacionado a la presentación de este trabajo. Dr. Perez relata apoyo no financiero de la empresa CLP, no relacionado a la presentación de este trabajo. Dr. Ruiz relata apoyo no financiero de la empresa CLP, no relacionado a la presentación de este trabajo.

Dr. Vergara relata apoyo no financiero de la empresa CLP, no relacionado a la presentación de este trabajo.

\section{Agradecimientos}

Quisiéramos agradecer a la Sociedad Chilena de Ortopedia y Traumatología por el apoyo en la obtención de las piezas cadavéricas para el presente estudio.

\section{Referencias}

1 Suh N, Grewal R. Controversies and best practices for acute scaphoid fracture management. J Hand Surg Eur Vol 2018;43(01):4-12
2 Dodds SD, Panjabi MM, Slade JF III. Screw fixation of scaphoid fractures: a biomechanical assessment of screw length and screw augmentation. J Hand Surg Am 2006;31(03):405-413

$3 \mathrm{Kim}$ RY, Lijten ECE, Strauch RJ. Pronated oblique view in assessing proximal scaphoid articular cannulated screw penetration.J Hand Surg Am 2008;33(08):1274-1277

4 Riddick AP, Hickey B, White SP. Accuracy of the skyline view for detecting dorsal cortical penetration during volar distal radius fixation. J Hand Surg Eur 2012;37(05):407-411

5 Klammer G, Dietrich M, Farshad M, Iselin L, Nagy L, Schweizer A. Intraoperative imaging of the distal radioulnar joint using a modified skyline view. J Hand Surg Am 2012;37(03):503-508

6 Jacob J, Clay NR. Re: Pichler et al. Computer tomography aided 3D analysis of the distal dorsal radius surface and the effects on volar plate osteosynthesis. J Hand Surg Eur. 2009, 34: 598-602. J Hand Surg Eur 2010;35(04):335-336

7 Capo JT, Orillaza NS Jr, Slade JF. Percutaneous Management of Scaphoid Nonunions. 2009;13(01):23-29

8 Levitz S, Ring D. Retrograde (volar) scaphoid screw insertion-a quantitative computed tomographic analysis. J Hand Surg Am 2005;30(03):543-548 